\title{
Determinants of Emergency Hospital Admissions among Patients in Blackpool, England: Population-Based Cross-Sectional Study
}

Gabriel Agboado ${ }^{1 *}$ and Judith Mills ${ }^{2}$

${ }^{1}$ Specialist Registrar, Public Health, NHS Blackpool, UK

${ }^{2}$ Public Health, NHS Blackpool, UK

\begin{abstract}
Objectives: To examine the relative importance of patients' socio-demographic characteristics, health service factors, health conditions, day and season of admission as predictors of emergency admissions among Blackpool residents.

Study design: Population-based cross-sectional study using routine patient admission data. Records of admissions among Blackpool patients were analysed to examine the effects of socio-demographic factors, health service factors, health conditions, day and season of admissions on emergency admissions.

Principal results: The emergency admission risk has declined over the study period. Compared with admissions in people aged 35 to 44 , admissions in those aged 85 and above were 3.2 times more likely to be emergencies. Admissions from 4 th IMD quintile areas were $43 \%$ more likely to be emergencies compared with those from $2 \mathrm{nd}$ IMD quintile areas. Admissions among patients registered with Blackpool GPs were less likely to be emergencies compared with those registered with GPs outside Blackpool. Admissions to geriatric medical specialties were most likely to be emergencies. Emergency admission risk was highest for admissions attributed to accidents. Emergency admission risk was also highest for Saturday admissions and lowest for Wednesday admissions. No significant association was observed between emergency admissions and patients' sex.
\end{abstract}

Conclusions: The risk of emergency admissions among Blackpool patients has declined over the period of this study. Socio-demographic and health service factors, health conditions, day and season of admission were independently associated with emergency admissions.

Keywords: Emergency hospital admissions; socio-economic deprivation; health conditions; health service factors

\section{Introduction}

Emergency hospital admissions have been increasing in the UK over recent years $[1,2]$. With the predicted increase of about $53 \%$ in the proportion of people aged 65 and over in the UK between 2001 and 2013 [3] this trend is set to continue as some evidence suggest that the ageing population structure will be associated with increases in the number and frequency of emergency admissions [4]. In addition to its impact on health service resources, hospitalisation can lead to adverse consequences particularly for older people, including breakdown of formal and informal care, loss of social networks, and premature admission to residential care [4].

A national audit of emergency admissions reported that the rising number of emergency admissions could cause problems in the organisation of care for patients admitted as emergencies and identified suboptimal involvement of consultants in acute care, frequent lack of appropriateness of the admitting specialty to patients' conditions, and confusion about policies for admitting elderly patients as significant problems [5]. Despite these concerns there is a paucity of evidence on relative importance of the various determinants of emergency admissions across all age groups and health conditions and reports on emergency admissions have tended to deal with hospital-based overall admission rates[1] or condition-specific admission rates [6-9].

The knowledge and understanding of the influences of the formal health system factors and patient attributes on the increasing trends in emergency admissions are important for identifying appropriate and effective interventions to manage the trend. The aims of our study are to describe the patterns of emergency admissions among Blackpool patients and examine the relative importance of some of the factors that influence emergency admissions.

\section{Method}

\section{Study population and setting}

Blackpool is a spearhead unitary local authority in Lancashire, the North West of England with an estimated population of 141,900 [10]. The proportion of those aged 65 and above in Blackpool (19.0\%) is significantly higher than the England (16.1\%) and the North West (16.4\%) averages [11].

There are 22 GP practices in Blackpool and in keeping with the wellknow practice across England, some Blackpool residents are registered with family doctors (GPs) outside Blackpool. The Blackpool Fylde and Wyre NHS Trust (BWFH) is the main provider of inpatient hospital care for Blackpool patients. Some patients use other acute trusts within and outside Lancashire because of proximity, choice or expertise not available at the BFWH. The Lancashire Care NHS Trust (LC) provides adult psychiatric care for Blackpool patients.

*Corresponding author: Gabriel Agboado, Public Health Department, NHS Blackpool, The Stadium Seasiders Way, Blackpool, FY1 6JX, UK, Tel: 44125365 1028; Fax: 44125365 1210; E-mail: gagboado@nhs.net

Received December 07, 2010; Accepted January 19, 2011; Published January 21, 2011

Citation: Agboado G, Mills J (2011) Determinants of Emergency Hospital Admissions among Patients in Blackpool, England: Population-Based CrossSectional Study. J Clinic Res Bioeth 2:107. doi:10.4172/2155-9627.1000107

Copyright: (c) 2011 Agboado G, et al. This is an open-access article distributed under the terms of the Creative Commons Attribution License, which permits unrestricted use, distribution, and reproduction in any medium, provided the original author and source are credited. 
The study used the Admitted Patient Care General Episode Commissioning Dataset for the period $1^{\text {st }}$ April 2005 to $31^{\text {st }}$ March 2009. This dataset normally covers all NHS and private provider admission spells in any hospital in England under the care of a consultant, midwife or nurse [12]. A provider spell is the time that a patient stays with one hospital care provider from admission to discharge, transfer or death [13]. Records of admission spells for Blackpool patients over the period were extracted for analyses.

Emergency admissions are admissions that are unpredictable and at short notice because of clinical needs [12]. Admissions classified as emergencies in this study were those coded as 21, 22, 23, 24 and 28 [12]. Primary diagnoses at admission were classified according to the World Health Organization's ICD-10 (International Classification of Disease, $10^{\text {th }}$ revision).[14] Specialty classifications used broadly followed those contained in the dataset [12] (see Appendix 1 for details). We used the ONS classification of the seasons used in the excess winter deaths calculations [15]. GP practices were coded $\mathrm{A}$ to $\mathrm{V}$ to eliminate the risk of identifying patients because of the relatively small practice list sizes.

Postcodes of patients' places of residence were linked to their respective 2007 Indices of Multiple Deprivation (IMD) [16] which are composite scores derived from seven deprivation domains. The scores were further grouped into their corresponding national score quintiles - the higher the quintile the more deprived an area is. The resultant dataset was analysed to describe emergency admission patterns and identify factors associated with emergency admission.

\section{Statistical analyses}

Emergency admission rates by the various factors of interest were calculated as a proportion of total admissions with their respective 95\% confidence intervals. Logistic regression analyses were carried out to identify and quantify any associations between the variables of interest (see Box 1) and emergency admission. Stats Direct version 2.7.7 was used for calculating the emergency admission rates with $95 \%$ confidence intervals while SPSS version 13.0 for Windows was used for all other analyses.

\section{Results}

\section{Emergency admissions}

There were 196,902 records of admissions among Blackpool patients over the period out of which 61,982 were classified as emergency admissions giving an overall emergency admission rate of $31.5 \%$. Majority $(68.2 \%)$ of the emergency admissions were via accident and emergency services (code 21), 20.1\% were via GP referrals (code 22), $0.4 \%$ were via bed bureau (code 23 ), $2.4 \%$ were via consultant outpatient clinics (code 24 ), while $8.8 \%$ were via other means including patients who arrived via the accident and emergency department of other healthcare providers (code 28).

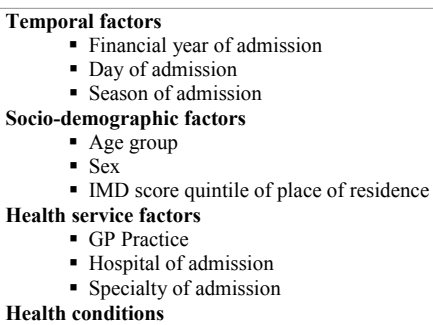

Box 1: Explanatory variables considered in the analyses.

\section{Temporal variations in emergency admission risk}

Emergency admission risk declined over the study period. The adjusted ORs indicate that compared with 2008/09 emergency admissions were about $7 \%$ more likely in $2005 / 06$ and $8 \%$ more likely in $2006 / 07$. There was no statistically significant difference in the risks for 2007/08 and 2008/09 (Table 1 provided as supplementary file).

The emergency admission risk varied by day of admission. The risk was highest on Saturdays and lowest on Wednesdays. Compared with admissions on Wednesdays, admissions on Saturdays were 9.0 times more likely to be emergencies, those on Sundays were 7.8 times more likely, those on Mondays and Fridays were 1.3 times more, those on Tuesdays were 1.2 times more likely while those on Thursdays were $8 \%$ more likely to be emergencies (Table 1 provided as supplementary file).

The results also show that emergency admissions were 5\% more likely between April and July compared with between August and November while there was no statistically significant difference between the emergency admission rates in December to March (the winter months) compared with August to November (Table 1 provided as supplementary file).

\section{Variations in emergency admission risk by socio-demographic factors}

Significant associations were observed between emergency admissions and patients' age. Admissions in those aged under 5 years were $18 \%$ less likely to be emergencies compared with admissions in those aged between 35 to 44 years, admissions in those aged 5 to 10 years and those aged 45 to 74 years were also less likely to be emergencies compared with admissions in those aged 35 to 44 years, while admissions in those aged 11 to 34 years and those aged 75 and above were more likely to be emergency admissions (Table 2 provided as supplementary file). There was no significant association between emergency admissions and patient's sex.

The results also show that the level of deprivation was associated with emergency admissions. For example, compared with admissions from the least deprived $2^{\text {nd }}$ IMD quintile areas, those from the most deprived $5^{\text {th }}$ quintile areas were $43 \%$ more likely to be emergencies while there was no significant difference between the emergency admission risks for $2^{\text {nd }}$ and $3^{\text {rd }}$ quintile areas.

\section{Variations in emergency admission risks by health service factors}

Variations in emergency admission risks by GP practice: Emergency admissions varied by patient's GP practice. Compared with admissions in Practice A patients, admissions in patients from 14 Blackpool GP practices were $13 \%$ to $59 \%$ more likely to be emergencies; admissions for patients from one Blackpool practice (Practice J) were 10\% less likely to be emergencies, while admissions in patients from practices outside Blackpool were 2.9 times more likely to be emergencies. The emergency admission risk was not significantly different for patients from 6 Blackpool practices compared with Practice A (Table 3 provided as supplementary file).

Variations in emergency admission risks by hospital factors: Emergency admissions varied by hospital of admission. Patients admitted to Lancashire Teaching Hospitals NHS Trust (LTH) were least likely to be emergencies while those admitted to LC were most likely to be emergencies. Admissions to LC were 23 times more likely to be emergency admissions compared with those to Lancashire Teaching 
Hospitals NHS Trust (LTH). Admissions to the BFWH were 5.6 times more likely to be emergency admissions, those to East Lancashire NHS Trust (ELH) were 5.2 times more likely, those to University Hospitals of Morecambe Bay NHS Trust (MBH) were 3.2 times more likely while those to hospitals outside Lancashire were $74 \%$ more likely to be emergency admissions compared with admissions to LTH (Table 4 provided as supplementary file).

Compared with paediatric surgical specialties, admissions to geriatric medical specialties were most likely to be emergencies followed by admissions to paediatric medical specialties. Admissions to all the other specialties were less likely to be emergencies based on both adjusted and unadjusted results expect for adult medical specialties and psychiatry for which adjustment reversed the association from positive to negative (Table 4 provided as supplementary file).

\section{Variations in emergency admission risks by health condition}

Health conditions influenced the likelihood of emergency admission. Compared with admissions attributed to diseases of genitourinary system, those attributed to accidents (i.e. injury, poisoning and certain other consequences of external causes) were 13.6 times more likely to be emergencies, those attributed to mental disorders were 11.0 times more likely to be emergencies, those attributed to infectious diseases were 4.3 times more likely to be emergencies, those attributed to respiratory conditions were 3.0 times more likely to be emergencies, those attributed to symptoms, signs and abnormal clinical and laboratory findings were 3.1 times more likely to be emergencies, those attributed to diseases of the nervous system were 1.3 times more likely to be emergencies while those attributed to circulatory system diseases were 1.2 times more likely to be emergencies. Admissions attributed to all other conditions including neoplasms, congenital malformations, digestive system conditions and musculoskeletal system diseases were significantly less likely to result in emergency admissions (Table 5 provided as supplementary file).

\section{Discussion}

\section{The findings in relation to other studies}

The results of this study indicate that emergency admission risks of have decreased over the study period and there were significant variations in the risk based on patients' characteristics, health service and temporal factors. The existing evidence suggests a continuing rise in emergency admissions $[2,17,18]$ with some suggesting that all age groups and diagnostic categories are involved in the rising trend with major contributions from older medical and surgical patients, multiple admissions, and socially deprived groups.[2] However Morgan et al have observed that the rise in emergency admissions may be attributable to the increased reporting of internal transfers of patients after admission [19]. The observed trend in this study may be due to several initiatives in Blackpool to improve the management of long-term conditions in primary care.

We found patients aged 75 years and above were most likely to experience emergency admissions. Hospital service utilisation is known to increase with age [4] highlighting the need for effective services to support this age group to reduce their emergency admission risks because the consequences of emergency admissions could be farreaching for them [4]. We also found that admissions in those aged between 45 and 74 years were less likely to be emergencies compared with those aged between 35 and 44 years suggesting the role of risktaking lifestyle factors in the latter age group. Patient's sex was not associated with emergency admission risk in this study whereas others have found the male sex to be associated with a higher emergency admission risk $[20,21]$. This apparent dissonance may be due to differences in the range of predictors considered, outcome measures, and study settings.

Our study also showed that emergency admission risks varied significantly by deprivation quintile. Admissions from the most deprived areas in Blackpool were about $43 \%$ more likely to be emergency admissions compared to those from the most affluent areas. The association between emergency admissions and socio-economic deprivation has been described particularly for cardiovascular and respiratory conditions [7,22-24], and some cancers [25] but may apply to other conditions not similarly investigated. However some have suggested that socio-economic deprivation may be a proxy for other factors such as ethnicity, level of social support and the quality of care received $[22,26]$ which may directly influence health outcomes.

Changes in population age and levels of morbidity, and social changes such as decreasing support for the elderly and increasing social deprivation, account for only a proportion of the increase in emergency admissions in the UK $[2,18,19]$ suggesting the role of other factors such as those within the formal health and social care system particularly in respect of managing long-term conditions [18]. We found that formal health service factors were significantly associated with emergency admissions among patients.

Blackpool patients registered with GPs outside Blackpool were almost two times more likely to experience emergency admissions compared with patients from most Blackpool practices. This finding may be a further evidence of the positive impact of initiatives within Blackpool primary care. Variations in GP referral practices [1,27] and quality of primary care [22] have been suggested by some as explanations for variations in emergency admissions.

We observed significant associations between emergency admission and hospital and speciality of admission. Admissions to the adult psychiatric hospital were associated with the highest emergency admission risk. This may be because adult psychiatry cases were mostly managed in the community with only acute exacerbations needing emergency admissions. The higher emergency admission risk observed for patients admitted by BFWH compared to other hospitals in Lancashire may be due to the proximity of patients to the hospital. Admissions to paediatric and geriatric medical specialties were also associated with the highest emergency admission risks. These two specialties manage the most vulnerable patient groups and admission policies might have been influenced by clinical uncertainties associated with managing these groups. Although these variations may also be manifestations of some of the issues identified by the emergency admissions national audit especially those relating to admission policies [5], a complex interplay between patient characteristics, referrers, availability of alternative forms of care and practices within hospitals have also been identified [28].

Predictably emergency admissions were most likely when patients presented to hospitals with accidents while patients with conditions associated with pregnancy, childbirth and the puerperium were least likely to experience emergency admissions reflecting the improved quality of antenatal and perinatal care in England generally. Accidents (excluding road traffic accidents and those associated with outdoor activities) are more common at the extremes of age. Accidental poisoning from medications may be more likely in older people with multiple chronic conditions on multiple drug therapies. Emergency admissions will tend to rise for these conditions as the population ages. 
Emergency admissions were most likely on Saturdays and least likely on Wednesdays in our study. Weekends are normally associated with low levels of elective admissions and anyone going to a hospital on weekends is more likely to be suffering from conditions requiring emergency hospital admission and treatment.

Existing evidence suggests that seasonal variations in emergency admission rates are condition specific with respiratory and cardiovascular conditions presenting more frequently during the winter $[17,22]$, and some especially surgical conditions tending to present more frequently in the summer [29], while other conditions show no seasonal variations [22]. Our study shows that emergency admissions were only $5 \%$ more likely in April-July compared with August-November while there was no significant difference between risks for August-November and December-March suggesting that the composite effect of the condition-specific variations have little effect on the overall seasonal variations.

\section{Implications for practice}

Factors influencing emergency hospital admissions among Blackpool patients were related to primary and secondary health service provision, patient characteristics and socio-economic deprivations. Interventions to reduce emergency admissions will need to take cognisance of these factors if they are to be effective. Interventions based on a shared understanding of these influences among primary and secondary care providers may be more successful.

Emergency admissions are a necessary point in the care pathway of older people and those with long-term and chronic conditions hence it is reasonable to expect them to increase in an ageing population as in the case of Blackpool. However effective identification and management of these patients with long-term conditions can avert some of the unplanned admissions. Practice based commissioning provides a good framework for developing services that support the older people and those with long-term conditions to prevent unplanned admissions, and for sharing good practices to ensure that the best practices are universal and accessible to all patient groups.

\section{Strengths, limitations and biases}

We use the Admitted Patient Care General Episode Commissioning Dataset for our study hence its main strength is the large number of records analysed to provide robust estimates of risks for a wide range of factors not usually considered together in a single study. Being an areabased study it provided a unique insight into the experiences of patients of all age groups, socio-economic background and with different health conditions, using different health care service providers.

Incompleteness and inaccuracy are the main problems associated with routine data sources. Even though there have been concerns about the quality of routine hospital data, data quality in the UK has improved greatly over the years [30]. The dataset we used should be accurate because it is one of the standard datasets used for performance monitoring, reconciliation and payments in the National Health Service and supports the implementation of the UK Department of Health payment by result policy [31].

All the records were complete for the factors investigated except for sex and health conditions for which only $0.01 \%$ and $1.01 \%$ respectively were missing. Though this level of missing data is unlikely to predispose the study to any selection bias, potential coding inaccuracies could lead to misclassification.

The factors included in the final model collectively explained about $56.3 \%$ (Nagelkerke R2 $=0.563$ ) of the variations in emergency admissions suggesting the role of other factors in the observed patterns of associations. Other likely predictors of emergency admissions e.g. comorbidities, severity of the primary diagnoses, and availability and quality of other health and social care services were not included in the regression models because they were not available in the dataset. We used very broad categorisations for many of the variables investigated (e.g. ICD 10 chapter headings for health conditions, age groups and treatment specialties) hence there is a potential for residual confounding.

We used the 2007 Index of Multiple Deprivation (IMD), a population level measure of deprivation, to assess the impact of deprivation on emergency admissions. Though IMD is widely used in studies to assess the impact of socio-economic deprivation on health outcomes [22-25] mainly because it is easily available, it could predispose to ecological fallacy as the associations at the population level may not necessarily represent associations at individual level [32].

\section{Conclusions}

The risk of emergency hospital admissions among Blackpool patients has declined over the period covered by this study. Age, socioeconomic deprivation, patient's GP practice, hospital and specialty of admission, health condition, day and season of admission were independently associated with emergency admission. Collectively these factors explained $56.3 \%$ of the variations in emergency admissions. Patient's sex was however not found to be associated with emergency admissions.

\section{Acknowledgement}

We are grateful to Mr. Jonathan Peters, Senior Public Health Analyst, NHS Blackpool, for retrieving the data and helping with processing the data for analysis.

Funding: The authors did not receive any funding for this work.

Ethical approval: The study was approved by the Clinical Quality Centre BFWH (Reference number: NT/SE0123). Ethical approval was not required for the use of the routine data.

\section{References}

1. Campbell WB, Lee E J K, Van de S K, Gooding J, Cooper M J (2002) A 25-year study of emergency surgical admissions. Ann R Coll Surg Engl 84: 273-277

2. Capewell, $S$ (1996) The continuing rise in emergency admissions. BMJ 312 991-992

3. Dr Foster Intelligence (2006) Keeping people out of hospital: The challenge of reducing emergency hospital admissions. London, Dr Foster Intelligence.

4. Walsh, B, Roberts, H C, Nicholls, P G, Lattimer, V A (2008) Trends in hospita inpatient episodes for signs, symptoms and ill-defined conditions: observational study of older people's hospital episodes in England, 1995-2003. Age Ageing 37: $455-458$

5. Houghton, A,Hopkins, A (1996) Acute medical admissions: results of a national audit. J R Coll Physicians Lond 30: 551-559

6. Gupta R, Sheikh A, Strachan D, Anderson HR (2003) Increasing hospita admissions for systemic allergic disorders in England: analysis of national admissions data. BMJ 327: 1142-1143

7. Hawker ال Babatunde, O, Farzana, S, Julius, W, Noel, G, Richard C W (2003) Social deprivation and hospital admission for respiratory infection: an ecological study. Respir Med 97: 1219-1224

8. Jordan RE, Hawker J I, Ayres J G, Adab P, Tunnicliffe W, et al. (2008) Effect of social factors on winter hospital admission for respiratory disease: a casecontrol study of older people in the UK. Br J Gen Pract 58: 400-402

9. Trotter CL, Stuart JM, George R, Miller E (2005) Increasing hospital admissions for pneumonia, England. Emerg Infect Dis 14: 727-733. 
Citation: Agboado G, Mills J (2011) Determinants of Emergency Hospital Admissions among Patients in Blackpool, England: Population-Based Cross-Sectional Study. J Clinic Res Bioeth 2:107. doi:10.4172/2155-9627.1000107

Page 5 of 5

10. Office for National Statistics (2009) Mid 2008 population estimates for UK London, Office for National Statistics.

11. Office of National Statistics (2009) Mid 2008 population estimates for UK.

12. NHS Data Model and Dictionary Service (2010) Commissioning Data Set. London, National Health Service.

13. Hospital Episode Statistics (2010) How do you spell that? London, The Health and Social Care Information Centre.

14. World Health Organisation (2010) International Statistical Classification of Diseases and Related Health Problems - 10th Revision, Version for 2007.

15. Office for National Statistics (2010) Winter Mortality. London, Office for National Statistics.

16. Social Disadvantage Research Centre Oxford University (2007) Indices of Deprivation 2007. London, Department of Communities and Local Government.

17. Afza M, Bridgman S (2001) Winter emergency pressures for the NHS contribution of respiratory disease, experience in North Staffordshire district. J Public Health 23: 312-313.

18. Kendrick S, Vize J (2007) Trends in rates of emergency inpatient admission among older people in Scotland: a comparative analysis at NHS Board level. Edinburgh, NHS Scotland.

19. Morgan K, Prothero D, Frankel S (1999) The rise in emergency admissions crisis or artefact? Temporal analysis of health services data. BMJ 319: 158-159.

20. Donnan PT, Dorward D W T, Mutch B, Morris A D (2008) Development and Validation of a Model for Predicting Emergency Admissions Over the Next Year (PEONY): A UK Historical Cohort Study. Arch Intern Med 168: 1416-1422.

21. Gorman J, Vellinga A, Gilmartin J J, O'Keeffe S T (2009) Frequency and risk factors associated with emergency medical readmissions in Galway University Hospitals. Ir J Med Sci 179: 255-259.

22. Damiani M, Dixon J (2002) Managing the Pressure: Emergency hospita admissions in London, 1997-2001. London, King's Fund.
23. Janghorbani, M, Jones, R B, Nelder, R (2006) Neighbourhood deprivation and excess coronary heart disease mortality and hospital admissions in Plymouth UK: an ecological study. Acta Cardiol 61:313-320.

24. Lyratzopoulos G, Havely D, Gemmell I, Cook G (2005) Factors influencing emergency medical readmission risk in a UK district general hospital: $A$ prospective study. BMC Emergency Medicine 5: 1

25. Raine R, Wong W, Scholes S, Ashton C, Obichere A, Ambler G (2010) Socia variations in access to hospital care for patients with colorectal, breast, and lung cancer between 1999 and 2006: retrospective analysis of hospital episode statistics. BMJ 340: b5479.

26. Bernard S, Smith L K (1998) Emergency admissions of older people to hospital: a link with material deprivation. J Public Health Med 20: 97-101.

27. Ingram JC, Calnan M W, Greenwood R J, Kemple T, Payne S, et al. (2009) Risk taking in general practice: GP out-of-hours referrals to hospital. $\mathrm{Br} \mathrm{J}$ Gen Pract 59: e16-e24.

28. Coast J, Peters T J, Inglis A (1996) Factors associated with inappropriate emergency hospital admission in the UK. Int J Qual Health Care 8: 31-39.

29. Ward ST, Mithen R J, Mohamed M S, Mufti G R (2009) Seasonal variation in emergency referrals to a Surgical Assessment Unit. Int J Clin Pract 63: 121 125

30. Audit Commission (2004) Information and data quality in the NHS. London, Audit Commission

31. NHS Connecting for Health (2010) Secondary Uses Service. London, National Health Service.

32. Memon A (2008) Epidemiological understanding: an overview of basic concepts and study designs. In Oxford Handbook of Public Health Practice. (Ed. D. Pencheon C. Guest, D. Melzer and M J A Gray) pp. 100-111, Oxford University Press, New York 